south it occurs at a lower elevation. The jet stream persists there also later into the polar day, as may be expected from the later heating of the antarctic stratosphere. It has not been possible so far to investigate synoptically the effect of the final collapse in spring of the polar night jet upon the circulation pattern in lower latitudes.

The scarcity of observations from the Southern Ocean area inevitably affected general circulation studies and the discussion of the interaction between the Antarctic and lower latitudes. In presenting his paper on antaretic synoptic analyses, W. J. Gibbs suggested that the presence and the main features of cyclones and anticyclones can be diagnosed by the analyst though there was still considerable doubt about the reality of frontal analyses. Synoptic studies of cold outbreaks and surges had been prepared by a number of Australian authors (A. K. Hannay, R. Falconer and H. M. Treloar, F. A. Berson and U. Radok). Circulation studies based on synoptic material were presented by J. Langford, I. S. Kerr, H. van Loon and S. Karelski.
Synoptic conditions over the antarctic ice cap itself are now reasonably well known for the first time. The communication system required to achieve this end was described by T. I. Gray, jun. It appears that cyclones do occasionally penetrate deep into the Antaretic and that fronts move sometimes right across the South Pole, though this occurs not as often as is probably the case in the north. In general, high-latitude cyclones seem to move sluggishly along the coast.

The happy international co-operation established between meteorologists in the Antarctic is perhaps symbolized by a paper submitted by P. D. Astapenko (U.S.S.R.) which was based on a joint investigation by him, J. Alt (France) and M. Ropar (United States). It is hoped that this co-operation will be continued in the International Antarctic Analysis Centre which is now being set up under the ægis of the Australian Bureau of Meteorology at Melbourne and that the work carried out there will lead to a better understand. ing of meteorological problems as a whole.

E. B. Kraus

\title{
HYDROGEN LIQUEFACTION
}

$\mathrm{T}$ HE use of liquid hydrogen, first produced and collected by Dewar at the Royal Institution in 1898, has, until recently, been largely confined to the laboratory. With a normal boiling point of $20.4^{\circ} \mathrm{K}$. it provides a convenient means of controlling the temperature for experiments in the range $14^{\circ}-90^{\circ} \mathrm{K}$. and it is used as a precooling agent in the liquefaction of helium.

On March 12, a one-day symposium on "Hydrogen Liquefaction and Associated Techniques", organized by the Low Temperature Group of the Physical Society, was held at the Institute of Physics in London, and it was ovident from the attendance at the meeting, as well as the topies discussed, that interest in the liquefaction of hydrogen on an industrial scale is developing in Great Britain.

The morning session, under the chairmanship of Dr. P. H. Sykes, was opened by Prof. G. O. Jones (Queen Mary College, London), who spoke of the applications of liquid hydrogen in pure research. He pointed out that it is necessary to go well below temperatures obtainable with liquid hydrogen in order to study the interesting features of many phenomena. Nevertheless, liquid hydrogen is of great technical assistance in many experiments. In optical spectroscopy, for example, the reduction in thermal motion of atoms at low temperatures results in a decreased Doppler broadening of spectral lines. The use of liquid hydrogen in bubble chambers, where it may form both a target and a detector for nuclear reactions, has also proved of great value.

In certain experiments, however, there is little point in working at temperatures below $20^{\circ} \mathrm{K}$. For example, the thermal expansion coefficients of most substances and variations in such properties of the solidified inert gases as the density, compressibility and refractive index become so small below $20^{\circ} \mathrm{K}$. that they are difficult to measure with precision. Such experiments may very conveniently be performed in miniature hydrogen liquefiers with about $100 \mathrm{ml}$. of liquid and in which liquefaction commences some 15 min. after starting at room temperature.

The design and construction of small-scale liquefiers were discussed by Dr. D. H. Parkinson (Radar
Research Establishment, Malvern), who emphasized the ease with which liquid hydrogen may be produced in the laboratory with relatively simple and inexpensive apparatus. High-pressure hydrogen, usually from a cylinder, is precooled with liquid nitrogen and then expanded at a valve into a reservoir. A proportion of the gas liquefies and the remainder is used to cool the incoming high-pressure gas by means of a heat exchanger between the liquid hydrogen and nitrogen stages. The yield of liquid depends rather critically on the pressure and precooling temperature ; for example, a standard $120 \mathrm{cu}$. ft. capacity cylinder exhausted from 120 to 50 atmospheres would yield 0.36 litre of liquid if the gas were precooled to $77^{\circ} \mathrm{K}$. and 0.55 litre with a precooling temperature of $65^{\circ} \mathrm{K}$. Impurities, usually amounting to about 0.1 per cent in commercial hydrogen, must be removed from the gas or they will solidify and block the liquefier tubes, and $150 \mathrm{gm}$. of activated charcoal cooled in liquid nitrogen was recommended as an adequate absorber for each cylinder of gas.

The use of liquid hydrogen as a source of 'cold' has already been mentioned, and an application to the production of a beam of low-energy neutrons was described by Dr. F. J. Webb (Atomic Energy Research Establishment, Harwell). Neutrons from a reactor with, say, a heavy-water moderator at a temperature of $50^{\circ} \mathrm{C}$. are thermal neutrons, that is, slow neutrons with energies of the same order as the thermal energies of atoms in a solid. They are spread over a range of values with the greatest number possessing energies corresponding to a de Broglie wave-length of about 1 A. In certain diffraction experiments, however, it is necessary to employ neutrons with wave-lengths greater than $4 \mathrm{~A}$., the number of which is relatively small compared with those of $1 \mathrm{~A}$. wavelength. Since the thermal energy is proportional to the absolute temperature, the kinetic energies of the neutrons may be reduced, and their wave-lengths correspondingly inereased, by using liquid hydrogen as a moderator. In the Dido reactor at Harwell, a small hydrogen liquefier feeds a 3-in. diameter container placed in the neutron beam and the liquid hydrogen moderator results in a gain of the order of 
25 in the flux of neutrons of the required wavelength.

Another application of liquid hydrogen to pure research was described by Mr. C. van Zyl (University College, London), who spoke of its use as a scattering target for high-energy particles. Interactions between two particles such as the proton and the neutron are being investigated as an approach to an understanding of the many-body problem. By employing three targets, one of liquid hydrogen, one of liquid deuterium, and a blank target to eliminate background scattering, it is possible to study $n-p, p-n$, $n-n$ and $p-p$ reactions in the same apparatus.

One of the difficulties encountered in building apparatus for low-temperature work is that of providing vacuum-tight windows for particles or radiation without eausing appreciable absorption or scattering. Mr. van Zyl described windows for the target chambers made of thin $(0.002$ in.) 'Terylene' film which will contain liquid hydrogen when the chambers are surrounded by a high vacuum.

As every schoolboy knows, the considerable size of a space rocket is largely accounted for by the quantity of fuel required to deliver a given load, and the search for more exotic fuels proceeds apace. The ultimate performance of a ballistic vehicle depends upon its 'burn-out' veloeity, and this is inversely proportional to the square root of the molecular weight of the fuel. 'Thus, the best rocket fuels have low molecular weights and are generally rich in hydrogen. From this aspect, liquid hydrogen is obviously a desirable choice, and in the afternoon session, under the chairmanship of Dr. M. Ruhemann, the possibilities were discussed by Mr. A. V. Cleaver (Rolls Royce, Ltd.).

It is estimated that combustion of hydrogenoxygen mixtures would increase the thrust by some 40 or 50 per cent over that obtainable with conventional kerosene and liquid oxygen fuels. Against this must be set the complications of accommodating a liquid of low boiling point and low density which would tend to reduce the quantity of propellant carried by the rocket. However, recent improvements in the construction of fuel tanks, so that they contribute to the structural rigidity of the rocket and reduce its unladen weight, would seem to make the use of liquid hydrogen more feasible. Although no work is being conducted in Britain, encouraging results have been achieved in the United States with small rocket motors using liquid hydrogen and liquid oxygen.

Clearly, if liquid hydrogen were successfully em. ployed as a fuel, the demand would exceed present production capacity very considerably. With this possibility in mind, Mr. W. L. Seddon (Petrocarbon Developments, Ltd.) presented a design study of a plant capable of producing 2 tons (about 30,000 litres) of liquid hydrogen por hour. This may be compared with one of the few large plants in existence, that at Boulder, Colorado, with a potential capacity of 600 litres per hour.

Hydrogen gas would be derived from oil refinery light hydrocarbon products, and since it is difficult to predict the future demand for liquid hydrogen the economics of the scheme were based on the production of heavy water by distillation. Liquefaction would take place by Joulo-Thomson expansion of compressed hydrogen, but one difficulty would be the removal of impurity, $\frac{1}{2}$ per cent of nitrogen present in the feed gas resulting in the deposition of $300 \mathrm{lb}$. of solid per hour. It was therefore proposed to precool the feed gas to about $35^{\circ} \mathrm{K}$. with the aid of expansion turbines and expand the gas from a pressure of about 16 atmospheres. Under these conditions, the efficiency of the Joule-Thomson process remains fairly high and a considerable proportion of impurity is carried through the expansion valve and deposited in the liquid reservoir, where it may easily be removed.

The capital cost of such a plant is in the region of $£ 6,000,000$, and the estimated price of the product, allowing for the sale of heavy water as a by-product, is $£ 200$ per ton. Liquid hydrogen produced on this scale would therefore work out at a cost of about 1s. a gallon, comparing surprisingly favourably with hydrocarbon fuels at, say, $4 s .8 d$. a gallon.

On a somewhat smaller scale, a paper by Dr. B. Shaw and Mr. W. H. Denton dealt with the problem of coping with a varying demand at the Atomic Enorgy Research Establishment, Harwell, by storing liquid hydrogen in 50-litre Dewar vessels. Hydrogen consists of molecules in two different rotational states, the equilibrium concentration being 75 per cent ortho-hydrogen and 25 per cent para-hydrogen, but the liquid is slowly converted to the para form with evolution of heat. In storage, therefore, liquid is lost by ortho-para conversion and it is usually more desirable to induce the conversion before liquefaction by passing the high-pressure gas over a eatalyst such as ferric oxide or activated charcoal at low temperatures. Dr. Shaw presented figures showing that, for short-term storage, greater overall economy is gained by earrying out partial conversion at $80^{\circ} \mathrm{K}$. than by converting completely to the para form at $20^{\circ} \mathrm{K}$. The total loss from a 50 -litre Dewar vessel with liquid nitrogen shielding may be as low as 0.18 litre per day.

For storage on a large scale, however, a vacuuminsulated vessel employing a liquid nitrogen radiation shield would be unduly large and complicated and the feasibility of using thermal insulation similar to that now employed in liquid nitrogen and oxygen storage vessels was discussed in a paper by $\mathrm{Mr}$. K. C. Smith and Dr. J. B. Gardner (British Oxygen Research and Development). In these vessels, the liquid is surrounded by a space evacuated to a pressure of $10^{-1} \mathrm{~mm}$. mereury and packed with an insulating powder. For liquid hydrogen, the loss from such a vessel would chiefly be due to radiation, and Mr. Smith described measurements of the overall thermal conductivity resulting from the addition of reflecting particles, such as aluminium powder, to the powders commonly in use. They show a significant decrease in thermal conductivity and indicate that the thickness of insulation need not be inconveniently great in, for example, a vessel of $10 \mathrm{ft}$. in diameter. Similar considerations are being applied to the design of transfer lines for large quantities of liquid.

The question of safety must be given prime consideration when dealing with liquid hydrogen, and some general remarks were made by Dr. A. J. Croft (Clarendon Laboratory, Oxford), who strossed the importance of making provision for possible human errors in operating liquefiers. Many of the precautions are common to any equipment containing low boiling-point liquids or gases at high pressure, as, for example, the fitting of relief valves to prevent the pressure rising above a safe value. One must also adopt the precautions normal when dealing with a highly inflammable liquid or explosive vapour, such as adequate ventilation of buildings. The con- 
sequences of a mishap should be minimized by partly enclosing liquefying plant by blast walls and by operating it remotely. This, of course, is not always feasible, particularly with small-scale laboratory installations.

Happily, there have been few serious accidents attributable to the use of liquid hydrogen, and reason- able and sensible safety precautions would appear to suffice. Judging by the papers and discussions presented at this symposium, it is conceivable that, in the future, liquid hydrogen will be produced and handled on a seale and with a facility comparable to that encountered to-day with liquid oxygen.

D. H. BOWEN

\section{PARASITOLOGY}

$\mathrm{T}$ HE first meeting outside London of the Parasitology Group of the Institute of Biology was held in the Zoology Department, University of Edinburgh, during March 19-21. It was the longest session so far held and the range of topics discussed was limited only by the general heading of "Parasitology".

Twenty-five papers were read, covering subjects as varied as the use of antigen-antibody reactions to differentiate species and strains of Entamoeba (V. Zaman), and the morphology, function and taxonomic status of the adhesive apparatus of Amphibdellatid parasites of electric rays (J. Llewellyn).

Some of the speakers were concerned with the mechanisms which trigger the changes in parasite life-histories. E. W. McConnachie reviewed the possibility that the onset of sexual reproduction of some parasites is related to hormonal changes in the host, and outlined her preliminary experiments with Opalina in frogs which tentatively support this view. Assuming a direct effect of the host's hormones on the parasite, one would have to postulate the presence of such hormones at the site of parasitism, which, for Opalina, is the lumen of the frog's rectum. Coccidian Protozoa would seem to be in a more favourable position to be influenced by systemie changes in the host since they develop in the epithelial tissues of the gut, but S. F. M. Davies showed that no mechanism of this kind was needed by Eimeria necatrix in chicks, the sexual phase of development being initiated by second-generation merozoites irrespective of the physiological state of the host.

The evolution of a temperature trigger mechanism in the life-cycle of certain tapeworms was argued by C. A. Hopkins on the basis of ecological studies on Proteocephalus filicollis in sticklebacks. He showed that in the north of Britain very few of the immature stages of these worms complete their development, even though they become successfully established in the fish host. Comparison with the state of affairs in the south suggested that low temperature can arrest development of such a species, limited, as it is, to cold-blooded hosts. An elegant solution to the parasite's problem would be the inclusion in the life-cycle of an 'incubator' in the form of a warm-blooded host. Hopkins suggested that this may have happened in the case of three-host pseudophyllidean tapeworms, since a rise in temperature is almost sufficient on its own to mature the plerocercoids of some of these parasites.

Mechanisms which enable free-living stages to locate their hosts were considered in two papers, one by C. A. Wright, the other by C. Ellenby and A. B. Gilbert. The former put forward evidence in favour of a response by trematode miracidia to substances exuded by their molluscan hosts. Ellenby and Gilbert presented a progress report on work with the hatching factor of potato root eelworm and showed that their results were still consistent with the view that an unsaturated lactone ring is diag. nostic of the molecule of the active constituent of potato root diffusate.

Problems more comprehensive than trigger mechanisms were considered by E. J. Bell, C. A. Hopkins and M. R. Wyllie. These workers described their joint efforts to bridge the gap between the analytical and synthetic aspects of the in vitro culture of strigeid metacercariae to the sexually mature adult fluke. Media synthesized from chemically defined and simple constituents were very limited in their ability to promote growth and had to be assessed by sensitive criteria, such as cell-division, rather than by an increase in size of the organism or its parts. On the other hand, some of the unknowns of complex media which do support considerable growth could now be replaced by known substances, but the gap between the two approaches was still formidable.

Experiments which illustrate the differences that may exist between the metabolism of host and parasite were described by L. P. Joyner. Both sulphadimidine and pyrimethamine on their own showed therapeutic effect when administered to chicks infected with Eimeria tenella, but the two administered together gave more than additive effect. This is consistent with the theory that they antagonize different links in the same metabolic chain of the parasite, namely, the $p$-aminobenzoic acid-folic-folinic acid sequence. The drugs are also toxic to the chick, but when they were administered, together with the metabolites they are presumed to antagonize, differential reversal of their effects on host and parasite occurred.

Investigations into mechanisms concerning resist. ance of hosts to their parasites were reported by $a$ number of speakers. F. W. Jennings presented the findings of a team of workers on immunity to Dictyocaulus infection in cattle. He showed that a double 'vaccination' of the host with irradiated infective larvæ protected it against further challenges of normal larvæ. L. Bailey dealt with the resistance of honeybees to epidemics of a range of parasites, and described how, without the aid of acquired immunity, the behaviour of the colony as a whole constituted a 'group immunity' reaction, and was successful in combating the disease as long as the community could develop normally. This was in part analogous to the dynamics of Sarcoptes scabiei infections in man, considered in two papers by $\mathrm{K}$. Mellanby and W. N. Boog Watson, though in this case acquired immunity was an additional factor. The breakdown of resistance to infection in 'resistant' strains of potato by eertain biotypes of potato root eelworm, and the investigation of its genetic basis in the nematode, was discussed by F. G. W. Jones. The evidence suggested a single locus for the effect. the double recessive conferring resistance-breaking, though the fact that some of the heterozygotes were resistance-breakers was against this simple interpretation. 